\title{
Spine Injuries in Sport
}

\author{
Stroe Alina Zorina ${ }^{1 *}$, Sirbu Carmen Adella ${ }^{2}$, Docu Axelerad Silviu ${ }^{3}$, Docu Axelerad Daniel ${ }^{4}$ \\ ${ }^{1}$ Department of Neurology, University Ovidius, Romania \\ ${ }^{2}$ Department of Neurology, University Carol Davila, Romania \\ ${ }^{3}$ Department of Neurology, Student at Vasile Goldis University, Romania \\ ${ }^{4}$ Department of Sport, Univeristy Ovidius, Romania
}

*Corresponding author: Stroe Alina Zorina, Department of Neurology, University Ovidius, Romania.
Received Date: April 15, 2020

Published Date: May 26, 2020

\begin{abstract}
The energy that is transferred through the collisions that occur in sports has the result of a number of critically harmed players every year. In the studies that have been made regarding to contact and noncontact sports it was showed that the most of sport related injuries of the spine are both soft-tissue injuries and self-limiting. The athletic injuries of the spine in a limited quantity have the outcome of significant neurological compromise. The result that appears after a soft tissue injury is the immobilization and the decrease of the performance. In the category of sports that are reported as „collision sports” are encountered the following: football, wrestling, and boxing, which can have results of acute traumatic lesions of the spine. These lesions have a distinct implication because of the common involvement of the cervical spine, that can cause a neurological deficit which targets all four members. Compressive type of stress injury is most commonly encountered in sports where the following elements are found: high body weight and important muscle contraction, such as football and weight lifting.The stress caused by the torsion appears in the throwing action, for example the javelin throwers, baseball players and golfers.
\end{abstract}

Keywords: Spine; Injury; Sport; Physical activity

\section{Introduction}

In particular, the promotion of sport is aimed at adolescents and young people with the aim of facilitating the harmonious development of the body and of discouraging obesity and its longterm consequences. In contrast, due to the exhaustive use of sports training in terms of intensity and volume, the practice of sports has conduced to an increased rate of acute and overuse injuries. The principal acute injuries in youth sports are sprains, strains, fractures, dislocations and contusions [1-5].

Young athletes are more prone to stress fractures and congenital predispositions to stress fractures. Young athletes can be affected by two basic types of injuries: acute injury as the result of single impact macrotrauma and overuse injury that appears after repetitive microtrauma of training.

The inconsistent variable that differentiates diagnosis, treatment and prognosis after spinal cord injury is related to the patient's age, due to the biomechanical and biochemical characteristics that each age.

\section{Discussion}

\section{intervertebral disc lesions}

Injuries can be disc-located with the result of disc herniation, disc degeneration, and ultimately developmental stenosis.These are most commonly seen in the lumbar spine L4-L5 and L5-S1, but also a significant incidence is present in the cervical spine. A low frequency of intervertebral disc lessions occurs at the level of thoracic spine. Disc lesions are generated by flexion/rotation or weightlifting injury, but also hyperextension can produce cervical disc herniation at the level of cervical spine C3-C4. Intervertebral disc lesions produce axial pain and restricted spinal motion. The clinical subjective symptoms of radicular compression are pain in a dermatomal distribution, weakness, paraesthesia and numbness. Neurological deficit is secondary to nerve root or spinal cord compression [6-10].

Compression injuries that use the axial loading process can result from injuries in sports using the motor or boat. It is possible 
to dislocate the fracture by rotation and flexion at the cervical and lumbar spine.

In any sport in which one athlete falls on another, an athlete may suffer an asymmetrical load and may suffer a rotation injury of the thoracolumbar spine. The intervertebral disc is frequently affected by rotational and shearing actions. Initially the affected structural layers can be separated or the inner layers may break. During the process of weakening and breaking the layers, forces are applied to the outer layers. This mechanism may cause radial rupture of the intervertebral disc. During the passage of time, the disc can heal, but with the appearance of the change of the capacity of biomechanical function [11-14].

In the case of an athlete who has a type of spinal obstruction, for example spinal stenosis, and who exercises in exercises involving extension maneuvers, they may increase the already present compression of the neurological structures and may cause local and clinical structural aggravation. In the case of an athlete who has a type of nerve root tension, for example, disc herniation, practicing exercises that involve flexion may produce increased tension in the nerve already under tension and may increase clinical symptoms. In the natural evolution of radiculopathy resulting from a herniation of the disc, the back pain evolves to the localized pain predominantly in the lower limbs. This pain presents aggravating factors through actions that increase intraspinal pressure, such as coughing, sneezing and sitting for a long time. At the objective examination, the patient shows positive signs of nerve stretching.

The source of the radicular pain that does not correspond to the aforementioned description is the radicular pain caused by spinal stenosis. Spinal stenosis is usually absent with signs of positive nerve stretching, but has the characteristic history of neurogenic claudication (ie, foot pain produced by walking). The pain does not cease at the moment of stopping and is aggravated by the extension of the spine, being improved by flexion. The pain progresses from proximal to distal. In conclusion, lesions characteristic of athletes may have presentations in the form of pain in the sacroiliac joint and pain in the posterior superior iliac spine.

The most prevalent area in which it manifests is the pain mentioned in the year of the intervertebral disc and the neuromotor segment of the spine. Compared to the population, the degradation of the lumbar intervertebral discs of the athletes is related to the movements that require the increased strength of the athletes, the excessive loading of the spine and the increased training time, and especially in the athletes the recurrence rate is high.

\section{Spondylolysis}

Spondylolysis is a stress fracture which is located in the pars intraarticular. It is most prevalent in sports which demants repetitive hyperextension and rotation. Fast bowlers in cricket, pitchers in baseball, gymnasts, tennis players and weightlifters are susceptible to develop this lesion type. Themost frequent location of spondylolysis and spondylolisthesis is L5-S1. The incidence of spondylolysis and grade I spondylolisthesis in sports participants is high. In the long term, this condition is not considered to be a significant factor in the inability of the athletes to perform.The patient has the development of the following clinical subjective symptoms: severe low back pain, in the first stage unilateral and then bilateral that aggravates with extension/rotation of the spine.

The basic mechanisms of the production of the injuries in sport activities produce a combined force vector. Three common mechanisms of injury that need to be taken into consideration are: (l) the mechanism of compression or loading of the spine by weight; (2) torque or rotation which may result in shear forces in a more horizontal plane and (3) the tensile tension produced by excessive movement of the spine.

Motion sports that use traction action on the spine include gymnastics, ballet, dance, pole vaulting, high jumping.Some injuries result from direct hits. Certainly, in sports, such as football, injuries are associated with muscle contusions, muscle extensions, and ruptures of the fascia, ligaments, and sometimes muscles. In gymnastics, spondylolysis can be present through the hyperextension action. In ballet the lumbar strain (arabesque position) and spondylolysis can occur. The lesions of weightlifters consist of compressive injuries. In football can occur: transverse process fractures, disc injury and contusion from the impact with the helmet. In runners, the back pain is prevalent. The golfers often present back pain and disc disease. In tennis the back pain can appear.

\section{Gymnastics}

In gymnastics, the location of the most frequent stress fractures is at the spine level for $42 \%$ of the cases, followed by the lower extremity location for $35 \%$ of the cases and the upper extremity location for $23 \%$ of the cases. The fractures that have the cause of stress are located at the lumbosacral spine are named spondylolysis. Gymnasts typically develop stress fractures in the upper lumbar spine and these fractures may be present at multiple levelsThis kind of fracture occurs frequently as a result of the repetitive spine's hyperextension, with the occurence mostly in: gymnastics, ballet, volleyball, diving and tennis.The gymnasts can present thoracic hyperkyphotic and lumbar hyperlordotic posture that predisposes these athletes to mechanical back pain. Studies have compared the incidence of spinal disc degeneration among elite and population gymnasts and revealed that the percentage of proffessional gymnast degeneration of the vertebral disc was $75 \%$, and the general population was $31 \%$.

\section{Aquatic sports}

In aquatic sports, the main motion of the spine concerns the rapid contraction modification of the lumbar paravertebral muscles through the rapid flexion and extension movements associated with the dive. Also, lesions of the cervical spine and those of the wrist are also associated. Even if swimming and water exercises are part of the back rehabilitation programs, there is the possibility, especially 
in young swimmers, to suffer certain injuries, in swimming types, such as butterflies, which produce a strong flexion / extension of the lumbar spine. In the pursuit of a proper swimming strategy, the swimmer must control the tone and strength of his abdominal muscles to be able to protect his back during a strong movement.

\section{Pole vaulting}

Pole vaulting follows the same pattern that includes a maximum action of flexion/extension and muscle contraction. The range of motion of the lumbar spine has been documented with high speed photography from 40 degrees of extension to 130 degrees of flexion in 0.65 seconds. The important forces that are generated across the spine demand a lot of functional charge.

\section{Weight lifting}

This type of physical activity requires muscular strength, lifting and high body weight. The most common such sport is weight lifting.Almost $40 \%$ of the weightlifters complain about the lower back pain. A compressive injury of the spine is the risk of the movements of athletes that lift weights above their head because of the important forces that affect the lumbar spine.The weight lifting process commences with the spine in narrow posture of flexion and the athlete lifts using the legs. Important force of extension is applied in the hips and knees locations with the spine in a firmly balanced position. This stage of the lift demands the body to produce tremendous rigid immobilization of the spine in the power posture of minor flexion.In athletes who lift weights, there is an almost constant increase in lumbar lordosis due to lifting weights above the level of the head. The use of these positions is related to the formation of important forces of extension of the lumbar spine that bring into question the possibility of spondylolysis and spondylolisthesis. The presence of spondylolysis in weight lifters has been estimated at 30\% and the incidence of spondylolisthesis has been estimated at $37 \%$. Due to the presence of these factors, new training methods have been created, which highlight the need to insist on the flexibility of the body as a whole, through the general conditioning of the body.

\section{Football}

Football is a sport where the risks of unexpected, severe contacts on the lumbar spine are present. The risk of contusion or fracture at any level of the spine are present too. In a paticular violent touch: the impact from a helmet that is applied to the ribs cand produce rib fractures. An impact applied on the flank has the possibility of production renal contusions, retroperitoneal hemorrhages, fractures located at the transverse and spinal processes.

\section{Running}

Running is the type of sport that produces rigidity. The need for constant improvement of flexibility is vital for long distance runners. During the run, the athlete must maintain a constant body posture with significant muscle exertion for a quantitative period of time. The most common painful places for athletes are: lumbar spine, cervical spine, interscapular space and shoulders.

\section{Golf}

Golfers have the highest predisposition and the highest frequency of injuries in the spine, among all professional athletes, due to the fact that the golf practice technique involves applying a significant torsional force to the lumbar spine.

\section{Javelin Throw}

The javelin throw consists of an important force that is generated by the athlete that must change the body position from a hyperextended position to a full flexion forward through position. The abdominal muscular strength and rigidity generates the torque that implies the javelin's throw. A injury in a javelin throw athlete through a rotatory lumbar spine injury is a totally incapacilitating injury that demands important care and correction.

\section{Baseball}

Torsional situations appear in pitchers and also in hitters. The throwers involve an important strength to be capable of transferring the force to the throwing arm. The strength of the trunk and the legs generates the throwing speed, and the arm ensures the fine resistance of control. Hitters need to initiate a violent lumbar rotation based on instantaneous ocular information and thus the role of the lumbar spine in the baseball bat begins with visualizing the ball. In case a hitter does not see the ball in time, the swing mechanism is changed.

The most common source of insults at this level is the observation of the late bile, which produces rotation with the hips in front of the shoulders, loss of parallelism of the shoulders and hips, and increased torsional tightening of the lumbar spine. The trunk should move fast as a solid unit, swinging the baseball bat.

\section{Conclusion}

Scientists support the theory that because training time, intensity and difficulty of performance athletes are close to the limits of the human body, the possibility of injury has increased greatly. In general, the incidence of lumbar disc herniation in athletes is much more prevalent than in the population, and at the onset of symptomatology, the degeneration of the detected disc is more advanced than that of the general public. Muscle strength, muscular endurance and muscle relaxation play an important role in preventing lumbar spine dysfunction. For example, the muscles of the posterior face of the legs originate from pelvic ischial tuberosity. In the event that these muscles are not optimally developed, they will apply forces that pull the posterior ischial tuberosity and will incline the posterior pelvis, forcing the lumbar spine to adopt a bent position by improper stretching of the back muscles, increasing the risk of protrusion of the cartilage of the intervertebral disc. Also, due to the optimal underdevelopment of the gluteal muscles and the tension in the back muscles, pelvic instability and back pain among athletes may occur.

The costs of the long-term health network for spinal cord injuries are a substantial economic complication. The effect from 
the social, but also economic point of view could be viewed from the point of view of the indirect costs of sports injuries such as absence from work and from the point of view of the long-term health repercussions, which are added to the costs. direct. An even moderate decrease in sports accidents is of great significance in the lives and health of athletes, especially young people and could have consequences on the long-term economic situation on healthrelated expenses. The addition of active prevention measures in the training methods of the athletes is necessary to increase the performance and reduce the risks of accidents and injuries. These measures should be considered as a whole multidisciplinary team made up of medical doctors, physical therapists, trainers and coaches, as well as the athletes themselves.

Studies conducted in the literature on the prevention of athletes' injuries through well-structured training programs have had beneficial results. Increasing endurance is more important than increasing muscle strength in proactive preventive management of athletes' injuries and, at the same time, increasing strength training of the back muscles, abdominal muscles and lower limb muscles to reduce the occurrence of back pain. The principle of beneficial exercise is to gradually increase the intensity and frequency, without causing pain. Reducing excessive repetitive movements must also be implemented, paying attention to the bending and turning movements, monitoring the intensity of the movements, strengthening the strength and basic softness of the muscles, and understanding the potential risk factors for player injury.

\section{Acknowledgement}

None.

\section{Conflict of Interest}

None.

\section{References}

1. Barnes C, Mercer G (2003) Disability (Cambridge, Polity).

2. Galli N, Vealey RS (2008) Bouncing back from adversity: Athletes' experiences of resilience. Sport Psychologist 22: 316-335.
3. Hall JM (2011) Narrative methods in a study of trauma recovery. Qualitative Health Research 21: 3-13.

4. Hicks AL, Martin KA, Ditor DS, Latimer AE, Craven C, et al. (2003) Longterm exercise training in persons with spinal cord injury: Effects of strength, arm ergometry performance and psychological well-being. Spinal Cord 41(1): 34-43.

5. Docu Axelerad A, Docu Axelerad D, Docu Axelerad S, Stroe AZ (2019) Walking in Parkinson's disease. Ovidius University Annals, Series Physical Education and Sport/Science, movement and health 19(2): 19 (2 supplement): 350-354

6. Stroe AZ, Docu Axelerad S, Docu Axelerad D, Docu Axelerad A (2019) Exercises in Parkinson's disease. Ovidius University Annals, Series Physical Education and Sport/Science, Movement and Health 19(2): 19(2 supplement): $344-349$.

7. Docu Axelerad A, Stroe AZ, Docu Axelerad S, Docu Axelerad D (2019) Divinity in dementia. Proceedings DIALOGO (The 10th Scholarly Meeting on the Dialogue between Science and Theology) 6(1): 187-194.

8. Docu Axelerad A, Stroe A Z, Docu Axelerad S (2019) Combating Depression in Parkinson's Disease with Melotherapy. Proceedings DIALOGO (The 10th Scholarly Meeting on the Dialogue between Science and Theology) 6(1): 195-202.

9. Docu Axelerad A, Stroe A Z, Docu Axelerad S (2019) How religiosity affects Parkinson's disease symptoms. Proceedings DIALOGO (The 10th Scholarly Meeting on the Dialogue between Science and Theology) 6(1): 203-211.

10. Dantes E, Docu Axelerad S, Stroe A Z, Docu Axelerad D, Docu Axelerad A (2020) The rehabilitation of hemiparesis after stroke. Ovidius University Annals, Series Physical Education and Sport / Science, Movement and Health 20(1): 5 -9.

11. Docu Axelerad A, Jurja S, Stroe AZ, Docu Axelerad S, Docu Axelerad D (2020) The role of physical exercise in multiple sclerosis. Ovidius University Annals, Series Physical Education and Sport / Science, Movement and Health 20(1): 10-15.

12. Docu Axelerad D, Docu Axelerad S, Dantes E, Stroe AZ, Docu Axelerad A (2020) Mixed dementia and physical exercise. Ovidius University Annals, Series Physical Education and Sport / Science, Movement and Health 20(1): 16-21.

13. Stroe AZ, Docu Axelerad S, Docu Axelerad D, Docu Axelerad A (2020) Neurorehabilitation through exercise in parkinson's disease patients, Ovidius University Annals, Series Physical Education and Sport /Science, Movement and Health 20(1): 67-71.

14. Docu-Axelerad A, Stroe ZA, Docu-Axelerad D, Docu-Axelerad S (2020) Multiple sclerosis and yoga. Arch Balk Med Union 55(1): 154-158. 